\title{
Adolescents with a learning disability
}

\author{
David Brooks and N. Bouras
}

The adolescents ( $n=57$, age 13-19 years) attending the special schools for moderate and severe leaming ditsabillities in a South East London health district and their fomilles were asesesed. The mothers of adolescents with severe learning disabilities in the transitional stage to adult services and development reported greater behaviour problems in thelr oftspring, greater stress, more negative psycho-social family effects and more negative aftitudes. Implications of the results for interventions, needs assesement and future research in family and adolescent functioning and the origins of psychlatic morblitty in young people with leaming disabilities wore discussed.

Community service issues and people with learning disabilities and their families are well illustrated as adolescents with a learning disability leave school and transfer to adult services. Researchers have used a variety of instruments to assess the social environment and family and child characteristics that contribute to stress and coping (Quine \& Pahl, 1989). However, as psychiatrists working in adult learning disability services in an urban area of South East London (population 320 000), we are aware of the lack of research, assessment, interventions and service planning for adolescents with learning disabilities at the time of transition to adult services. This is reflected in patterns of referral to our service.

\section{The study}

The aim of this study was to assess maternal stress; the burden of care of having a disabled adolescent in the family; family coping mechanisms; the degree of learning disability, medical and behaviour problems in adolescents, and factors related to the adult transition in order to define areas for service intervention and evaluation. The total number of adolescents ( $n=57$, age range 13-19) attending the four schools for moderate and severe learning disabilities in the district were included in the study. The adolescents whose transition to adult services was more imminent (17-19 years) and the relatively younger (13-16 years) were compared. Semistructured interviews were conducted with the class teacher and the mother.
The assessment procedure included:

Malaise Inventory - to measure levels of stress experienced by mothers

Problem Inventory - to assess the psychosocial effects on parents of raising a disabled child

Judson Self-Rating Scale of Maternal Attitudes - to measure maternal adjustment and resource in parenting

Family Support Scale - to assess the degree to which different sources of professional and informal support are helpful to families rearing a child with a learning disability.

For further details of the measures, and the self devised aspects of the assessment the reader is referred to the original report (Brooks \& Bouras, 1991).

\section{Findings}

Adolescents with a learning disability

Descriptive characteristics

The sample consisted of $31(54 \%)$ males and 26 (45\%) females. The age range was 13-19 years with 35 adolescents $(61 \%)$ in age group 13-16 years and $22(39 \%)$ in age group $17-19$ years. A moderate learning disability (DSM-III-R criteria) was present in 43 adolescents $(77 \%)$ and a severe learning disability in 14 (23\%). The cause of learning disability was as follows: Down's syndrome 15 (26\%), perinatal brain damage $5(9 \%)$, chromosomal defect 2 (3\%), other causes 14 (25\%) and not known 21 (37\%). Regular medication was prescribed for $16(28 \%)$ of adolescents; $81 \%$ of whom were receiving anticonvulsants for epilepsy. A physical disability was present in 22 (39\%) and an incapacitating medical disorder in $10(18 \%)$ of adolescents.

Behaviour problems

A behaviour problem was present in 34 adolescents (59\%). Common presentations included aggression, self-injury and inappropriate sexual behaviour. There were no statistically significant differences between the frequency and severity of 
behaviour problems in the younger and older age groups. When age and degree of disability were considered, the frequency and severity of problems were greater in the severely disabled group. The differences were statistically significant for the older group ( $t$ test $2.2 P<0.01, t$ test $2.0 P<0.05$ respectively).

\section{Burden of care, stress and family coping mechanisms}

Malaise Inventory. The mean score for mothers was 4.8 ( $\geq 5$ abnormal). Maternal stress was greater in mothers of the severely disabled with highest scores in the mothers of the older (transitional) age group (mean=6.2).

Problem Inventory. The study highlighted the more consistently high and more negative scores for 'extra time demands', 'life centering around their offspring', and 'need to constantly supervise and watch'. The mean score of 8 of the 10 measures (range 1-5) showed the highest and most negative effects in families with an older severely disabled member (extra time demands mean $=4.5$, life centering around their offspring mean $=4.0$, need to constantly supervise and watch mean $=4.0$, special family problems mean=3.5, $t$ test $2.6 P<0.5$ ).

Judson Scale. The mean score for mothers was 116.4 (range 23-161). Higher scores are associated with more positive attitudes. Mothers of the younger moderately disabled adolescents had the most positive attitudes (mean=117.8). The most negative attitudes were seen in mothers of the older, more severely disabled group (mean=105.5).

Family Support Scale. Perceived social support was poor in our population of mothers (mean score 23.9 range $0-88$ ) with a negative attitude to professionals (mean score 8.2 range 0-28). Higher scores are associated with more positive attitudes to support.

A correlation analysis of the independent variables was undertaken to study the possible inter-relationships of the adolescent and family characteristics. An association of greater frequency and severity of behaviour problems in the families of the older adolescents and extra time demands $(r=0.7, P<0.01)$, and greater pressure of care $(r=0.8, P<0.001)$ was found. Excessive care demands in the younger age group correlated with maternal stress $(r=0.5$, $P<0.01)$. Mothers of the younger age group with positive attitudes registered less negative family effects: less pressure of care $(r=0.6$, $P<0.001)$, more time for siblings ( $r=0.5, P<0.01$ ), and less disruption of family routine $(r=0.5$, $P<0.01$ ).

\section{Comment}

The study has clearly demonstrated that the mothers of adolescents with severe learning disabilities in the transitional stage to adult services and development reported greater behaviour problems in their offspring, greater stress, more negative psycho-social family effects and more negative attitudes. The importance of long term excessive direct care demands, behaviour problems and severe degree of learning disabilities of adolescents in the genesis of maternal stress and ability to cope is suggested. Excessive care demands at an early age might also contribute to the development of maternal stress.

The complicated medical needs and high prevalence of behaviour problems in adolescents with learning disabilities are a major issue for adult medical and psychiatric services. Our original report identified significant unmet needs, a lack of early interventions and questioned the targeting and effectiveness of current services (Brooks \& Bouras, 1991). This was also reflected in mothers' perception of professional social support. None of the adolescents with behaviour problems in this study had been seen previously by the child psychiatric services. This is pertinent to recent recommendations for people with learning disabilities, mental health needs and challenging behaviour (SETRHA. 1993).

Health commissioners, including GP fundholders and providers, have specific responsibilities in assessing the needs of this population and must play an active role in joint agency planning and the co-ordination of services. This should be considered in the context of community care planning mechanisms with other agencies including educational and vocational to ensure a seamless transition to adult services. Health professionals have a responsibility to implement effective communication systems to co-ordinate work between different provider units (Department of Health, 1989).

The assessment procedure employed in this study represents a composite of objective and subjective assessment methods for adolescents with a learning disability and their families. In order to successfully address needs assessment and to determine objectives (i.e. to reduce the prevalence of behavioural problems or psychiatric morbidity in adolescents with learning disabilities or to improve family coping) it is necessary to define the function of the services and indicators of input, process and outcome. Clinicians must strive to further develop valid, reliable and more comprehensive and easily administered assessment procedures.

The following variables are suggested for consideration in the development of such a process: 
(a) behaviour disorders, multiple disabilities, degree of learning disability of adolescents

(b) maternal stress and resources, psychosocial family effects

(c) service interventions focused at an early stage and at the transitional period to adult services

(d) social support.

Future research in family and adolescent assessment and needs identification must also include paternal considerations, personality factors and life events. Longitudinal studies beginning in early development are essential to facilitate this process, and to obtain a better understanding of family functioning and the origins of psychiatric morbidity in adolescents with learning disabilities.

\section{Acknowledgements}

We are grateful to the adolescents and their families who participated in the study, and to Marion Hair.

\section{References}

BROOKS, D. \& BOURAS. N. (1991) Assessment and Services for Adolescents with a Mental Handicap and Their Families. Division of Psychiatry, Guy's Hospital, London. DEPARTMENT OF HEALTH (1989) The Children Act 1989. London: HMSO.

QUINE, L \& PAHL. J. (1989) Stress and Coping in Families Caring for a Child with Severe Mental Handicap: a longitudinal study. Institute of Social and Applied Psychology and Centre for Health Service Studies, University of Kent at Canterbury.

SETRHA (1993) The Mental Health Needs of People With Learning Disabilities. Report of a Working Group. Bexhill: South East Thames Regional Health Authority.

David Brooks, Consultant Psychiatrist; and N. Bouras, Consultant Psychiatrist, Division of Psychiatry, Section of Learning Disabilities, United Medical and Dental Schools, Guy's Hospital, London SE1 9RT

\section{Corrigenda}

In the article 'Winston Churchill Travelling Fellowship to USA to Study Trauma Services for Children by Dora Black (Psychiatric Bulletin, September $1994,18,565-568$ ) the captions of the photographs of the Eric Lindemann Mental Health Center, Boston, Harvard University and Professor Pynoos' office at UCLA were transposed.
The address at the end of the paper 'A view from the road: experiences in four continents' by Vikram Patel (Psychiatric Bulletin, August 1994, 18, 500-502) should read: Department of Psychiatry, University of Zimbabwe Medical School, PO Box A178, Avondale, Harare, Zimbabwe. 